\title{
Correlação Anatomoclínica \\ Caso 4/2004 - Morte súbita em mulher de 24 anos portadora de arterite de Takayasu (Instituto do Coração do Hospital das Clínicas - FMUSP, São Paulo)
}

\author{
Sandra Falcão, Dante Marcelo Artigas Giorgi, Desidério Favarato, Luiz Bortolotto, \\ Paulo Sampaio Gutierrez \\ São Paulo, SP
}

Mulher de 24 anos de idade apresentou cansaço e dispnéia desencadeada por esforços aos 14 anos de idade, quando foi feito o diagnóstico de tuberculose, e recebeu tratamento específico.

Aos 19 anos de idade, no 30 trimestre da gravidez, apresentou hipertensão arterial de difícil controle e edema de membros inferiores. 0 parto foi na 38a semana e a pressão arterial persistiu elevada.

Aos 23 anos de idade, voltou a apresentar dispnéia desencadeada por esforços moderados, cefaléia frontal latejante e episódios de escurecimento da visão. No exame clínico, foi detectada a diminuição dos pulsos arteriais nos membros inferiores.

A investigação, com o uso de arteriografia, revelou artérias subclávia direita e carótida direita com múltiplas irregularidades, artéria carótida interna esquerda afilada, e suboclusões de artérias carótida externa e vertebral esquerdas. As porções descendente e abdominal da aorta apresentavam irregularidades e tortuosidades. A artéria renal direita revelou obstrução proximal estimada em $70 \%$, havia irregularidades em artéria renal esquerda e oclusão das artérias ilíaca interna e externa direitas, e suboclusão da artéria ilíaca esquerda. Foi feito o diagnóstico de arterite de Takayasu e prescritos prednisona e metotrexate e, para tratamento da hipertensão, anlodipino.

Os exames laboratoriais $(1 / 3 / 00)$ revelaram $9,4 \%$ de hemoglobina, hematócrito $29 \%, 6100$ leucócitos/ $\mathrm{mm}^{3}$; ácido úrico $4,4 \mathrm{mg} / \mathrm{dL}$; triglicérides $74 \mathrm{mg} / \mathrm{dL}$, colesterol $101 \mathrm{mg} / \mathrm{dL}$; glicose $85 \mathrm{mg} / \mathrm{dL}$; creatinina $0,8 \mathrm{mg} / \mathrm{dL}$; sódio $137 \mathrm{mEq} / \mathrm{l}$ e potássio $4,1 \mathrm{mEq} / \mathrm{L}$. 0 exame de urina foi normal.

A radiografia de tórax (2/3/00) revelou área cardíaca normal, aorta alongada e parênquima pulmonar normal.

Houve bom controle da pressão arterial até que em abril de 2000 , quando em nova gravidez, a hipertensão voltou a ficar fora de controle. A gravidez foi complicada por sangramento devido a descolamento prematuro de placenta e foi interrompida com curetagem uterina. Apesar da interrupção da gravidez a pressão arterial continuou de difícil controle.

Foi internada (10/5/00) para controle da hipertensão arterial, que era 190/110 mmHg; fazia uso de $10 \mathrm{mg}$ de anlodipino, $20 \mathrm{mg}$ de enalapril e $50 \mathrm{mg}$ de hidroclorotiazida e $5 \mathrm{mg}$ de

Editor da Seção: Alfredo J osé Mansur - E-mail: ajmansur@ incor.usp.br Editores Associados: Desidério Favarato

E-mail: dclfavarato@ incor.usp.br

Vera Demarchi Aiello - E-mail: anpeva@incor.usp.br

Correspondência: Paulo Sampaio Gutierrez - InCor - Av. Dr. Eneas de Carvalho Aguiar, 44 - 05403-000 - São Paulo, SP

182 E-mail: anppaulo@incor.usp.br amilorida, e $60 \mathrm{mg}$ de prednisona. Foi reduzida a dose de prednisona para $30 \mathrm{mg}$ e houve controle adequado da hipertensão.

0 eletrocardiograma $(10 / 5 / 00)$ revelou ritmo sinusal, freqüência de 100 bpm e sobrecarga ventricular esquerda (fig. 1).

As catecolaminas séricas estavam dentro dos limites normais. A aldosterona estava elevada, 35,6 ng/dl (normal em decúbito até $16 \mathrm{ng} / \mathrm{ml}$ ).

A ressonância magnética do abdome (maio/2000) revelou aorta abdominal com irregularidades parietais difusas e ausência de estenose significativa. 0 tronco celíaco tinha estenose acentuada na porção proximal; a artéria mesentérica superior apresentava estenoses acentuadas e dilatações saculares nos segmentos proximal e médio. A artéria renal direita, estenose moderada em porção proximal e dilatação irregular da porção média. As artérias ilíacas eram ectasiadas e irregulares com estenose intensa da ilíaca interna esquerda e oclusão da externa direita na origem, com presença da colateral proeminente das lombares. Os rins eram de dimensões normais.

Dois meses após (3/7/00) apresentou edema agudo dos pulmões. Inicialmente atendida em outro hospital, com a melhora do quadro foi transferida para o Pronto-Socorro do InCor.

0 exame físico (3/7/00) revelou pressão arterial de 170/80 mm $\mathrm{Hg}$, freqüência cardíaca de 80 bpm; estertores crepitantes em bases pulmonares, ritmo cardíaco regular, sopro sistólico em foco aórtico e sopro em carótidas. Havia, também, sopro sistólico em mesogástrio. Os pulsos pediosos, tibiais posteriores, popliteos e femoral direito estavam ausentes.

O ecocardiograma (7/7/00) revelou espessura de septo interventricular e de parede posterior de $13 \mathrm{~mm}$, diâmetros diastólico e sistólico de ventrículo esquerdo $49 \mathrm{~mm}$ e $33 \mathrm{~mm}$, fração de ejeção de ventrículo esquerdo 0,69; átrio esquerdo $32 \mathrm{~mm}$, aorta $35 \mathrm{~mm}$, ventrículo direito $19 \mathrm{~mm}$; a motilidade das paredes ventriculares era normal.

A ultra-sonografia de julho/2000 revelou bexiga normal. Os parênquimas renais estavam normais. Havia pequeno grau de uretero pielocaliectasia à direita e mínima ectasia da pelve renal esquerda. Foram observados dois pequenos pontos hiperecogênicos em grupos caliciais superior e médio à direita que podiam corresponder a diminutos cálculos. 0 rim direito mediu $11,6 \mathrm{~cm}$, e o esquerdo $12,5 \mathrm{~cm}$ (eixo longitudinal).

A cintilografia miocárdica perfusional com MIBI-Tec $99 \mathrm{~m}$ (7/7/00) demonstrou captação homogênea do traçador e foi considerada negativa para isquemia miocárdica; a função sistólica do ventrículo esquerdo estava preservada e havia aumento da espessura miocárdica. 
Foram prescritos $100 \mathrm{mg}$ de atenolol, $20 \mathrm{mg}$ de enalapril, $50 \mathrm{mg}$ de hidroclorotiazida, $20 \mathrm{mg}$ de anlodipino, $200 \mathrm{mg}$ de ácido acetil-salicílico, $30 \mathrm{mg}$ de prednisona diários e $10 \mathrm{mg}$ de metotrexate semanais.

Procurou atendimento médico novamente em 14/9/00 por dor lombar direita, febre $\left(38,5^{\circ} \mathrm{C}\right)$ e artralgias. A creatinina foi $1,2 \mathrm{mg} /$ $\mathrm{dL}$, a uréia $34 \mathrm{mg} / \mathrm{dL}, 13,1 \mathrm{~g} / \mathrm{dL}$ de hemoglobina, hematócrito $41 \%$, volume corpuscular médio $77 \mu \mathrm{m}^{3}, 18300$ leucóticos $/ \mathrm{mm}^{3}$ (4\% bastões, $81 \%$ segmentados, $9 \%$ linfócitos, $6 \%$ monócitos), 370000 plaquetas $/ \mathrm{mm}^{3}$, 3,4 mEq/L de potássio e $140 \mathrm{mEq} / \mathrm{L}$ de sódio.

Foram feitos os diagnósticos de infecção urinária e hipertensão renovascular por arterite de Takayasu. Recebeu ceftriaxone $2 \mathrm{~g}$ diários por 15 dias, com melhora do quadro de lombalgia e febre.

Nova ultra-sonografia (setembro/2000) revelou rins tópicos, de forma, contornos, dimensões, espessura do parênquima e textura normais. Foi diagnosticado cálculo calicial de $0,46 \mathrm{~cm}$ no grupo calicial inferior do rim direito; notou-se pequena dilatação do sistema coletor à esquerda pela presença de cálculo de $1,0 \mathrm{~cm}$ no ureter proximal a $4,5 \mathrm{~cm}$ da pelves renal. 0 rim direito media cerca de $11,3 \mathrm{~cm}$, e o esquerdo, $13,6 \mathrm{~cm}$ (eixo longitudinal). A bexiga estava normal.

Recebeu alta hospitalar, contudo duas semanas após apresentou parada cardiorrespiratória no domicílio e foi trazida ao InCor. As manobras de reanimação não alcançaram sucesso.

Discussão do eletrocardiograma (fig. 1) - As diretrizes da Sociedade Brasileira de Cardiologia sugerem os seguintes critérios para diagnóstico de sobrecarga ventricular esquerda: índice de Sokolov (S de V1+R de V5) $\geq 35 \mathrm{~mm}$ ou índice de Cornell ( $R$ de $\mathrm{aVL}+\mathrm{S}$ de $\mathrm{V} 3$ ) $\geq=28 \mathrm{~mm}$ em homens e $\geq 20 \mathrm{~mm}$ em mulheres; tempo de inscrição da deflexão intrinsecóide ou tempo até pico de onda $\mathrm{R} \geq 50 \mathrm{~ms}$; alteração de repolarização em derivações esquerdas (I, aVL V5 e V6): onda T achatada (valor na fase precoce) ou padrão tipo strain (infradesnivelamento do segmento ST de convexidade superior e T negativa assimétrica). Presença de sinais indiretos de sobrecarga, quando houver sobrecarga de átrio esquerdo.

O eletrocardiograma da paciente revelou índices de Sokolov e Cornell acima dos limites normais, sem alteração de repolarização ventricular ou evidências de sobrecarga atrial esquerda, o que sugere sobrecarga ventricular esquerda sem intensa hipertrofia miocárdica.

(Dr. Desidério Favarato)

Na aortografia por ressonância magnética (fig. 2), a aorta abdominal apresenta acentuadas irregularidades parietais difusas e um pequeno aneurisma acima das artérias renais. Além disso, são observadas oclusões do tronco celíaco e da mesentérica inferior e graves lesões em ambas artérias ilíacas. A imagem da arteriografia confirma essas lesões, além de mostrar lesões aneurismáticas e segmentares de carótidas e de subclávia esquerda, onde há lesão estenótica significante. Essas lesões são características da arterite de Takayasu, por acometerem a aorta abdominal, grandes vasos e artérias do arco aórtico.

\section{(Dr. Luiz Bortolotto)}

Aspectos clínicos - 1) Trata-se de paciente jovem, do sexo feminino, com quadro clínico inespecífico; as suspeitas diagnósticas, segundo esse perfil epidemiológico, são doenças infecciosas, doenças do colágeno e vasculites.
O quadro de dispnéia e cansaço apresentado aos 14 anos de idade e diagnosticado como tuberculose poderia ser questionado, tendo em vista que esses sintomas não são comuns na tuberculose não complicada, isto é, sem derrame pleural ou forma miliar. Há relação, ainda pouco definida, entre a tuberculose e a doença de Takayasu. Embora a etiologia da arterite de Takayasu permaneça desconhecida, existe questionamento sobre a possibilidade de agentes infecciosos agirem como desencadeadores ou gatilhos; entre esses, o Mycobacterium tuberculosis. Algumas evidências tanto diretas como indiretas sustentam esta hipótese. Entre as evidências diretas estão o fato da lesão de ambas as doenças serem granulomatosas, diferindo apenas pela presença ou não do bacilo, a reatividade cutânea ao PPD que ocorre em até $47 \%$ dos paciente com Takayasu e da relativa freqüência, até $30 \%$, da associação de tuberculose ativa e da arterite ${ }^{1}$. Além disso, o aumento dos níveis séricos de anticorpos contra Mycobacterium tuberculosis dá suporte indireto à relação entre os processos patológicos.

A doença de Takayasu, também conhecida como doença sem pulso ou coarctação reversa, tem sua distribuição ubíqua; no entan-

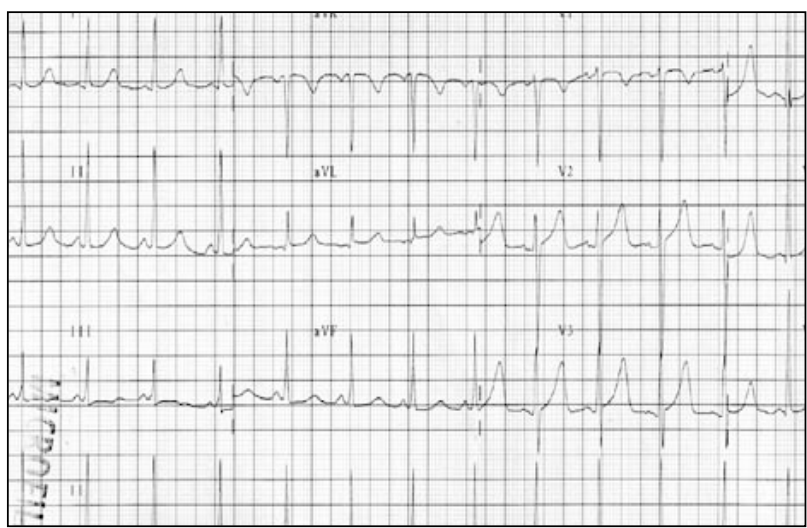

Fig. 1 - Eletrocardiograma - sobrecarga ventricular esquerda.

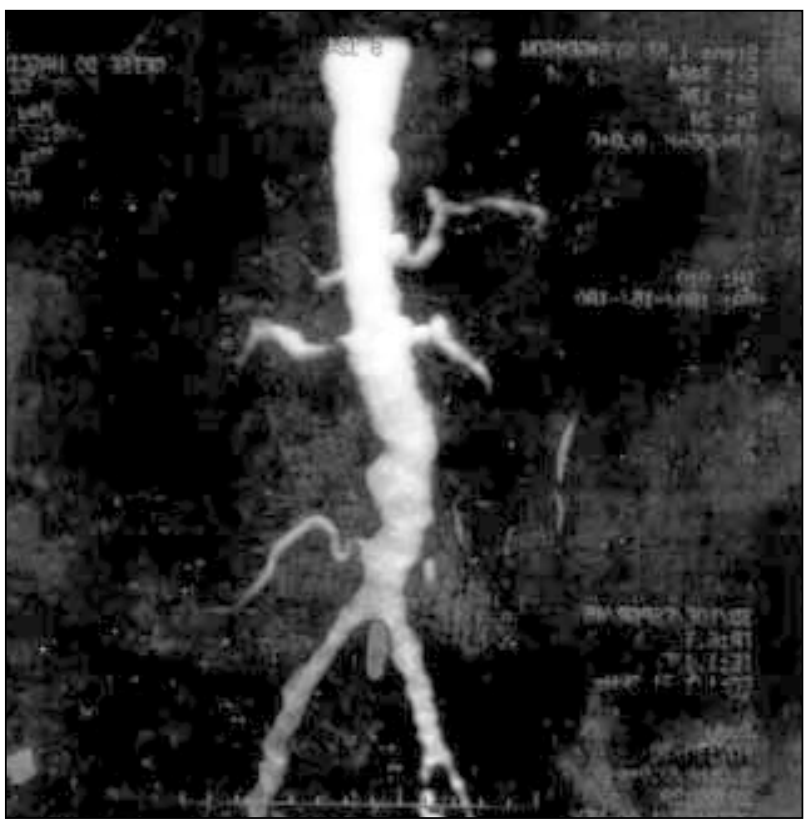

Fig. 2 - Aortogafia por ressonância magnética. Irregularidades aórticas difusas, oclusão de tronco celíaco e mesentérica inferior. Lesão suboclusiva de renal direita e lesões mais distais em renal esquerda. Artérias ilíacas acentuadamene acometidas, mais grave à direita. 
to, há maior prevalência nos países asiáticos e América central. Trata-se de uma doença inflamatória crônica que acomete as três camadas da parede de vasos de médio e grande calibre, principalmente aorta e seus ramos proximais.

As mulheres são mais freqüentemente afetadas, na proporção de 8:1 homem, e a média de idade ao diagnóstico é de 29 anos; o tempo médio entre o início das sintomas e o diagnóstico varia entre 2 e 11 anos ${ }^{2}$.

A doença é marcada por períodos de atividade e recrudecência com fases inflamatórias e cicatriciais. As manifestações clínicas dependem da fase da doença, estando inicialmente presentes sintomas inflamatórios inespecíficos, como febre, sudorese noturna, perda de peso, cefaléia, eritema nodoso, artralgias e mialgias. Posteriormente, surgem sintomas secundários aos processos cicatriciais (estenoses), como claudicação de membros, principalmente os superiores, amaurose fugaz e ataques isquêmicos encefálicos transitórios.

Ao exame físico destaca-se a ausência de pulsos, principalmente em membros superiores. Os sopros são freqüentes, estando presentes em $80-94 \%$ dos casos. A regurgitação aórtica, por dilatação da aorta ascendente, é observada em 20 a 24\% dos pacientes ${ }^{2}$. A hipertensão arterial encontra-se comumente presente, sendo em sua maioria de causa renovascular. A insuficiência cardíaca pode aparecer como resultado da hipertensão ou, mais raramente, da insuficiência aórtica.

A paciente em questão apresentou aos 19 anos, ao final da gestação, quadro de hipertensão de difícil controle, que persistiu mesmo após semanas do parto. Aos 24 anos nova gravidez gerou novo descontrole dos níveis pressóricos. A hipertensão é a complicação cardiovascular mais comum na gravidez, perfazendo 8-10\% de todas as gestações e importante causa de morbimortalidade maternofetal. A diferenciação entre hipertensão crônica e hipertensão gestacional (ou doença hipertensiva específica da gestaçãoDHEG) é baseada no fato de que na DHEG a hipertensão é induzida pela gravidez, surgindo após a $20^{\text {a }}$ semana de gestação e desaparecendo em até $6^{a}$ semana após o parto.

A arterite de Takayasu não altera a fertilidade e a gestação por si só não exarceba a doença. Entretanto, a presença de DHEG ocorre em cerca de $60 \%$ dos casos e, em pacientes já hipertensas, o descontrole da pressão é o maior complicador da gestação ${ }^{3}$.

0 diagnóstico de arterite de Takayasu é baseado em critérios clínicos e angiográficos, sendo um dos mais difundidos o da American College of Rheumatology que possui sensibilidade em torno de $77 \%$ e especificidade de $95 \%$, sendo necessária a presença de três dos seis critérios seguintes: idade $<40$ anos no início dos sintomas, claudicação de extremidades, diminuição de pulso braquial, diferença de pressão arterial sistólica > $10 \mathrm{mmHg}$ entre os braços, sopro em subclávia ou aorta e anormalidades angiográficas ${ }^{4}$. Na paciente citada, o quadro clínico levou à suspeita diagnóstica, confirmada tanto com base nos critérios clínicos como pelas alterações angiográficas.

Os exames laboratoriais são inespecíficos, podendo apresentar anemia de doença crônica, PPD positivo, elevação de VHS e proteína $C$ reativa, marcadores de atividade da doença, e sinais de sobrecarga ventricular esquerda ao eletrocardiograma.

0 tratamento é feito com corticosteróide e metotrexate, sendo que nos casos mais graves a doença cicatricial progride a tensão, aneurismas e insuficiência aórtica são preditores de pior prognóstico.

A causa de morte geralmente está associada a complicações cardiovasculares: isquemia miocárdica devido a acometimento coronariano tanto por estenoses, aneurismas ou embolias, ou cerebrais: microembolismos, tromboses ou isquemia por baixo fluxo sangüíneo ${ }^{5}$.

No caso em estudo, os exames complementares realizados no período de março a setembro/2000 sugerem progressão da doença com sítios vasculares cada vez mais seriamente acometidos apesar do tratamento, e as características clínicas da paciente, incluindo-a em grupo de pior prognóstico. 0 quadro febril de setembro/2000 poderia ser interpretado, tanto como um processo infeccioso quanto como período de atividade da doença, embora este último fosse pouco provável tendo em vista a dose alta de corticosteróide e imunossupressor prescritas.

(Dra. Sandra Falcão)

Hipóteses diagnósticas - A morte súbita da paciente deve ter sido causada por doença: cardiovascular: infarto agudo miocárdio com fibrilação ventricular primária ou acidente vascular cerebral por isquemia ou ruptura de aneurisma.

(Dra. Sandra Falcão)

Discussão clínica - 2) A paciente em discussão apresenta 0 diagnóstico de hipertensão renovascular unilatral por arterite de Takayasu. 0 diagnóstico foi suspeito pela presença de alterações clínicas sugestivas de doença arterial sistêmica (ausência de pulsos em artéria braquial, sopro abdominal e sopro carotídeo), em mulher jovem (23 anos), da raça negra, com hipertensão arterial grave desde os 19 anos de idade, e confirmado pela arteriografia que evidenciou estenose de artéria renal direita, além das outras lesões vasculares já descritas ${ }^{6,7}$. A hipertensão renovascular é a principal causa potencialmente curável de hipertensão arterial, principalmente na população mais jovem, isto é, abaixo de 20 anos. Nessa população, apesar de prevalência ainda alta de hipertensão primária, as causas secundárias são mais freqüentes do que na população adulta com idade superior, $(20 \%$ vs. $5 \%)$, respectivamente. Desta forma, é imprescindível investigação mínima de causa secundária para a hipertensão arterial em pacientes jovens, idade $<20$ anos ${ }^{8}$. Esta investigação mínima inclui dados da história clínica como uso de medicamentos que elevam a pressão arterial (corticosteróides, anticoncepcionais orais, descongestionantes nasais, anorexígenos), sintomas sugestivos de feocromocitoma (tríade de palpitações, sudorese e cefaléia), de coarctação de aorta (claudicação intermitente) ou de hipopotassemia por hiperaldosteronismo (câimbras, etc). Além disso, no exame clínico devemos avaliar assimetria de pulsos, presença de sopro abdominal ou outras características de doenças endócrinas. Se na história ou no exame físico não houver evidências de uma eventual causa, sugere-se a realização de rastreamento básico para as principais causas secundárias: sedimento urinário e creatinina sérica (nefropatia), cintilografia renal ou Doppler de artérias renais ou angiorressonância de artérias renais (renovascular), dosagem de renina e aldosterona plasmáticas (hiperaldosteronismo) e dosagem de metanefrina urinária (feocromocitoma). Na nossa paciente, o diagnóstico de hipertensão renovascular por arterite de Takayasu foi realizado pela arteriografia renal. Em nosso meio e, principalmente em países 
asiáticos, a arterite de Takayasu é uma causa importante de estenose de artéria renal, atingindo em nossa experiência a prevalência de $11 \%$, superior à da displasia fibromuscular ${ }^{9}$. Nessas pacientes, como na paciente em discussão, a hipertensão arterial é grave com repercussões renais e cardíacas importantes ${ }^{8}$. Em geral, acomete mulheres jovens, principalmente crianças e adolescentes. Em nossa experiência, envolvendo 45 pacientes, mais da metade dos casos tinha idade $<20$ anos de idade, a maioria com envolvimento de múltiplas artérias e com insuficiência cardíaca grave. A resposta ao tratamento corretivo da estenose da artéria renal ou da aorta quando presente promove controle mais adequado da pressão arterial e melhora da insuficiência cardíaca na maioria dos pacientes, principalmente nas crianças. No entanto, em alguns casos mais graves, mesmo após a correção cirúrgica, e com o tratamento com corticóides ou imunossupressores, a doença evolui com acometimento de vários órgãos e a morte acontece por complicações cerebrovasculares ou por infarto do miocárdio ${ }^{8}$. A paciente não realizou o tratamento corretivo da estenose de artéria renal, pois encontrava-se em evidente atividade inflamatória da doença, e nesses casos existe alta taxa de insucesso no procedimento, além de alta morbidade. Assim, optou-se pelo tratamento da atividade com corticóides e imunossupressor para, posteriormente, realizar a correção da estenose. No entanto, a paciente evoluiu com piora das lesões vasculares e morte súbita, apesar do tratamento adequado.

\section{(Dr. Luiz Bortolotto)}

Discussão Clínica - 3) Qual o diagnóstico diferencial etiológico da hipertensão arterial sistêmica em paciente jovem do sexo feminino?

Além dos diagnósticos já discutidos pelo Dr. Bortolotto, devemos considerar, em pacientes com quadro de pré-eclâmpsia, com proteinúria e que persiste após o parto, o diagnóstico de nefropatia primária. Cerca de $50 \%$ das pacientes com eclâmpsia e préeclâmpsia são portadoras de nefropatia primária. A gravidez, em muitos casos, favorece a piora de função renal dessas pacientes, principalmente nas glomerulopatias do lúpus eritematoso sistêmico. O exame sumário de urina, a pesquisa de proteinúria e a dosagem plasmática de uréia e creatinina devem ser solicitados na avaliação de pacientes jovens com quadro de hipertensão gestacional.

(Dr. Dante Marcelo Artigas Giorgi)

\section{Necropsia}

À necropsia, a principal doença encontrada foi arterite crônica inespecífica com intensidade variável, destruição de fibras elásticas, densas áreas de fibrose e espessamento fibrointimal (fig. 3) quadro morfológico que, apesar da ausência de células gigantes, leva ao diagnóstico de arterite de Takayasu. $\mathrm{Na}$ aorta, havia grande espessamento da parede, com aterosclerose secundária focal, formação de pequeno aneurisma e áreas de estreitamento da luz vascular (fig. 4). As artérias renais e a mesentéricas estavam também comprometidas, bem como segmentos das coronárias. Nestas, foram observados aneurisma abrangendo do $3^{\circ}$ ao $5^{\circ}$ centímetros do ramo interventricular anterior da artéria coronária esquerda, e acometimento leve, sem inflamação importante, e com espessamento fibrointimal discreto da artéria coronária direita.
No terço medial desta última, havia coágulo não-oclusivo (fig. 5). É provável que corresponda a trombo bastante recente, mas seu aspecto morfológico deixa um pouco de dúvida quanto à possibili-

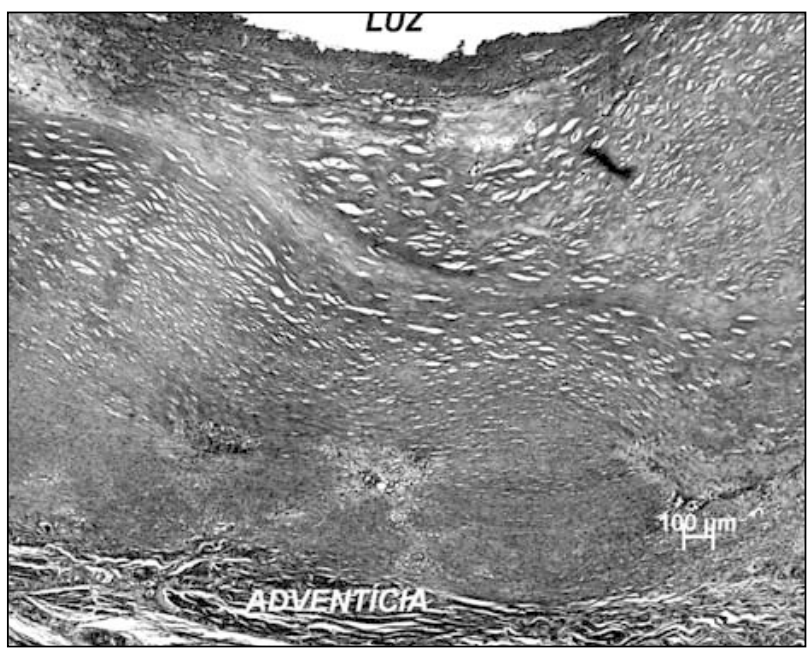

Fig. 3 - Corte histológico da aorta mostrando densa fibrose (em azul) da parede da artéria. (tricrômico de Masson, objetiva de $5 x$ de aumento).

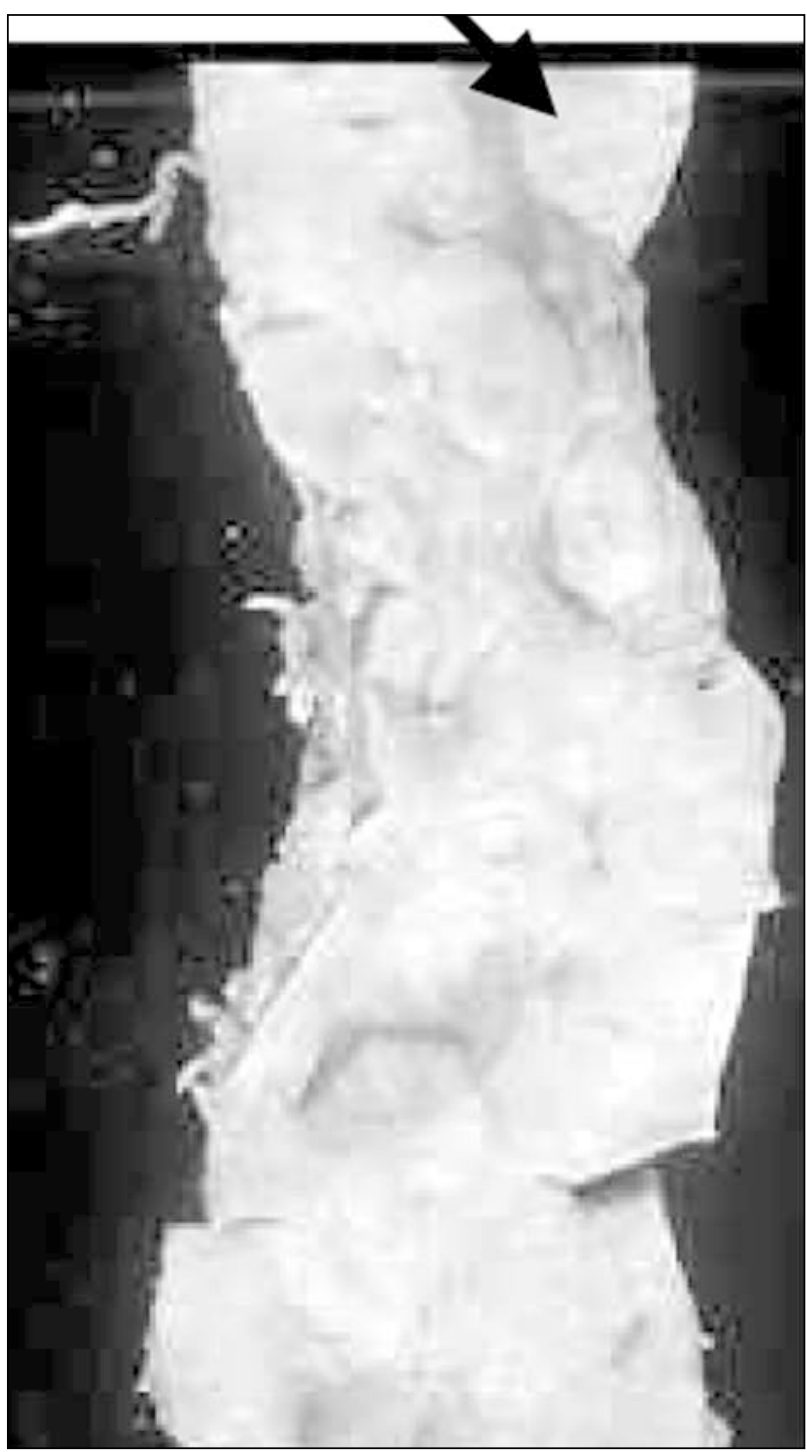

Fig. 4 - Aorta descendente aberta longitudinalmente, observada em sua face intimal. A artéria está espessada, com muitas irregularidades, área de aneurisma (seta) e com aterosclerose secundária. 
dade de que se trate de coágulo post-mortem. 0 coração, por sua vez, tinha hipertrofia concêntrica do ventrículo esquerdo, decorrente de hipertensão renovascular determinada pelas lesões em artérias renais, mas não alterações características de infarto antigo ou recente. De toda forma, embora infelizmente não se possa ter certeza com relação qual foi a causa mortis, pois não houve achados irrefutavelmente positivos, que explicassem o óbito, 0 mais provável é que tenha ocorrido trombose de artéria coronária e infarto do miocárdio, cujo período de evolução seria curto e assim insuficiente para determinar as alterações morfológicas características (se o paciente não sobrevive pelo menos 2 a $4 \mathrm{~h}$ após infarto do miocárdio, é difícil detectá-lo morfologicamente) ${ }^{10} \mathrm{De}$ todo modo, é interessante notar que o acometimento dessa artéria era relativamente discreto, não havendo processo inflamatório semelhante ao da aorta. Fica sem explicação o motivo que levoua à sede da trombose.

Ao que tudo indica, a dilatação que define os aneurismas da aorta decorre da ação de enzimas que destroem componentes do sistema elástico da camada média arterial ${ }^{11}$. Tais enzimas, das famílias das serino e metaloproteases, são liberadas principalmente pelas células inflamatórias. Torna-se interessante que na arterite de Takayasu, a despeito da inflamação, que inclusive parece ser acompanhada de elevação de níveis séricos de algumas metaloproteases ${ }^{12}$, ocorra redução da luz arterial. Assim como outros aspectos da etiopatogênese da doença, os mecanismos que modulam essa resposta predominantemente constritora nela presente não são ainda conhecidos.

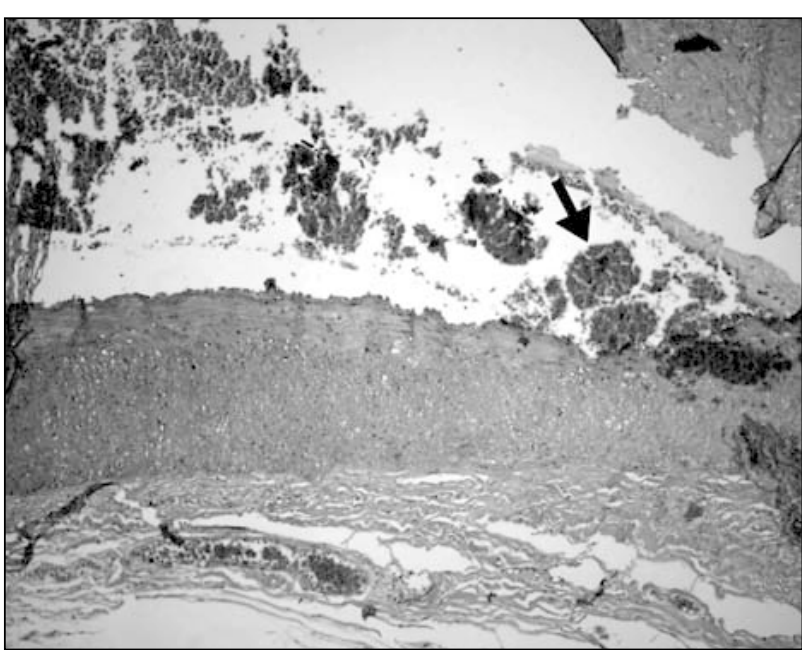

Fig. 5 - Corte histológico da artéria coronária direita em seu terço medial. Notase a presença de trombo não-oclusivo (seta), cuja adesão à parede é duvidosa. (hematoxilina e eosina, objetiva de $10 x$ de aumento).

Em outros órgãos, a única alteração vista foi dilatação do ureter esquerdo, associada à litíase urinária de que a paciente era portadora.

\section{(Dr. Paulo Sampaio Gutierrez)}

Diagnósticos anatomopatológicos - Doença principal: arterite de Takayasu; provável causa mortis: infarto do miocárdio.

(Dr. Paulo Sampaio Gutierrez)

\section{Referências}

1. Sheikhzadeh A, Tettenborn I, Noohi F, Eftekharzadeh M, Schnabel A. Occlusive Thromboaortopathy (Takayasu Disease): Clinical and angiographic features and a brief review of literature. Angiology 2002; 53: 29-40.

2. J ohnston SL. Takayasu arteritis: a review. J Clin Pathol 2002; 55: 481-6.

3. Langford CA, Kerr GS. Pregnancy in vasculitis. Curr Opinion Rheum 2002; 14: 36-41.

4. Neidhart B; Kosek R, Bachman LM, Stey C. Exertional dyspnea as initial manifestation of Takayasu's arteritis - A case report and literature review. BMC Pulm Med 2001; 1: 3

5. Franco FGM, Giorgi DMA, Giorgim MCP, Gutierrez PS. Clinico pathologic Session: The patient is an 11-year-old girl with Takayasu's arteritis and heart failure (Institute do Coração do Hospital das Clínicas/FMUSP - São Paulo) Arq Bras Cardio 2001; 77: 587-91.

6. Pariser KM. Takayasu's arteritis. Current Opinion in Cardiology. 1994; 9: 575-580
7. J ain S, Sharma N, Singh S, Bali HK, Kumar L, Sharma BK. Takayasu Arteritis in children and young Indians. Int J Cardiol 2000; 75: S153-S7.

8. Arap S, Denes FT, Bortolotto L. Renovascular hypertension in children. Curr Urol Rep 2001; 2: 181-2.

9. J ohnston SL, Lock RJ , Gompels MM. Takayasu arteritis: a review. J Clin Pathol 2002, 55: 481-6

10. Vargas SO, Sampson BA, Schoen FJ . Pathologic detection of early myocardial infarction: a critical review of the evolution and usefullness of modern techniques. Mod Pathol 1999; 12: 635-45

11. Ailawadi G, Eliason J L, Upchurch J r GR. Current concepts in the pathogenesis of abdominal aortic aneurysms. J Vasc Surg 2003; 38: 584-8.

12. Matsuyama $A$, Sakai $N$, Ishigami $M$, et al. Matrix metalloproteinases as novel disease markers in Takayasu arteritis. Circulation 2003; 108: 1469-73. 